\title{
Synthesis of a Cationic Polyacrylamide under UV Initiation and Its Flocculation in Estrone Removal
}

\author{
Jiaoxia Sun $\mathbb{D}^{1,2}$ Xiqin Ma $\mathbb{D}^{1,2}$ Xiang Li, ${ }^{3}$ Jianxin Fan, ${ }^{1,2}$ Qingkong Chen, \\ Xuelian Liu, ${ }^{1,2}$ and Jin Pan ${ }^{1,2}$ \\ ${ }^{1}$ School of River and Ocean Engineering, Chongqing Jiaotong University, Chongqing 400074, China \\ ${ }^{2}$ Key Laboratory of Hydraulic and Waterway Engineering of the Ministry of Education, and National Engineering Research Center for \\ Inland Waterway Regulation, Chongqing, China \\ ${ }^{3}$ School of Civil Engineering and Architecture, Chongqing University of Science and Technology, Chongqing 401331, China \\ Correspondence should be addressed to Jiaoxia Sun; sjx2008cqjt@126.com and Xiqin Ma; xiqin_ma5917@126.com
}

Received 7 September 2017; Accepted 16 November 2017; Published 1 February 2018

Academic Editor: Robert A. Shanks

Copyright (c) 2018 Jiaoxia Sun et al. This is an open access article distributed under the Creative Commons Attribution License, which permits unrestricted use, distribution, and reproduction in any medium, provided the original work is properly cited.

\begin{abstract}
A ternary cationic polyacrylamide (CPAM) with the hydrophobic characteristic was prepared through ultraviolet- (UV-) initiated polymerization technique for the estrone (E1) environmental estrogen separation and removal. The monomers of acrylamide (AM), acryloyloxyethyl-trimethyl ammonium chloride (DAC), and acryloyloxyethyl dimethylbenzyl ammonium chloride (AODBAC) were used to synthesize the ternary copolymer (PADA). Fourier transform infrared spectroscopy (FT-IR), ${ }^{1} \mathrm{H}$ nuclear magnetic resonance spectroscopy ( ${ }^{1} \mathrm{H}$ NMR), thermogravimetry/differential scanning calorimetry (TG/DSC), and scanning electron microscopy (SEM) were employed to characterize the structure, thermal decomposition property, and morphology of the polymers, respectively. FT-IR and ${ }^{1} \mathrm{H}$ NMR results indicated the successful formation of the polymers. Besides, with the introduction of hydrophobic groups (phenyl group), an irregular and porous surface morphology and a favorable thermal stability of the PADA were observed by SEM and TG/DSC analyses, respectively. At the optimal condition $(\mathrm{pH}=7$, flocculant dosage $=4.0 \mathrm{mg} / \mathrm{L}$ and $\mathrm{E} 1$ concentration $=0.75 \mathrm{mg} / \mathrm{L}$ ), an excellent E1 flocculation performance (E1 removal rate: $90.1 \%$, floc size: $18.3 \mu \mathrm{m}$, and flocculation kinetics: $22.69 \times 10^{-4} \mathrm{~s}^{-1}$ ) was acquired by using the efficient flocculant PADA-3 (cationic degree $=40 \%$, and intrinsic viscosity $=$ $6.30 \mathrm{dL} \cdot \mathrm{g}^{-1}$ ). The zeta potential and floc size analyses were used to analyze the possible flocculation mechanism for the E1 removal. Results indicated that the charge neutralization, adsorption, and birding effects were dominant in the E1 removal progress.
\end{abstract}

\section{Introduction}

Water is regarded as one of the most essential resources for the survival, daily life, and production activity of humans. However, the water pollution and contamination have severely restricted and hindered the development of national economy and society. Meanwhile, the water pollution and contamination also pose potential threat to wildlife and human health. Recently, the environmental problem caused by endocrine disrupting chemicals (EDCs) contamination has become the discussion focus of the public and regulatory agencies due to its harmful and hazardous effects to wildlife and human health [1]. The EDCs with the structural and functional similarity as entogenous estrogen can alter the functions of the endocrine systems and consequently cause adverse health effects to an intact organism, or its progeny or (sub) populations [2]. Most seriously, the EDCs have potential harmful impacts on the hormonal control, sexual differentiation, reproductive success, and community structure of aquatic organisms and their offspring even at extremely low concentrations [3]. It was reported that the EDCs such as the natural estrogens $17 \beta$-estradiol (E2) and estrone (E1), synthetic estrogen $17 \alpha$-ethynylestradiol (EE2), and industrial compounds of nonylphenol (NP) and propylparaben (PP) have been widely detected and checked in surface freshwater, marine water, landfill leachate, and sewage sludge [4]. Therefore, it is urgently needed to develop green and efficient water treatment technologies to remove and separate EDCs from water. 
Based on the above perniciousness analysis of EDCs, a number of technologies have been used to remove EDCs, and these technologies mainly include adsorption, biological methods, membrane treatment, and advanced oxidation processes [5-8]. Although these methods have a good performance on the removal of EDCs, the higher energy consumption, higher operation cost, cumbrous equipment, and complex running procedures hinder their further application in the waste water treatment. Fortunately, the flocculation technology has obtained more and more attention in waste water purification and separation due to its easy operation, high efficiency, low cost, and environment-friendly method [9]. As a result, this technology has been widely and extensively used in separating and dewatering solid/water system. So far a lot of flocculants with different functions have been developed and used in the suspended solids removal, sludge dewatering, dye wastewater treatment, humic acid removal, and Escherichia coli removal [10-14]. Therefore, it is proposed to use the flocculant for the EDCs separation and removal.

Cationic polyacrylamide (CPAM) is one of the most widely applied flocculants for the separation of colloidal suspensions. The CPAM with the positive charge can neutralize the negatively charged colloidal particles completely, and hence the stabilization of the colloidal particles is destroyed and disrupted [15]. Then, the destabilized colloidal particles are wrapped and connected to form large flocs and fast settle down under the effect of bridging and sweeping. Generally speaking, flocculation of fine particles can occur by polymer bridging, charge neutralization, polymer-particle surface complex formation, or their combination. The acrylamide (AM), acryloyloxyethyl-trimethyl ammonium chloride (DAC), and hydrophobic acryloyloxyethyl dimethylbenzyl ammonium chloride (AODBAC) are the commonly used monomers with no damage to the environment. Besides, the AODBAC with the hydrophobic groups (phenyl group) will cause the systems with unique rheological characteristics, thus leading to the formation of transient networks and the increase of solution viscosity. These associations result in an increase in the reaction between hydrophobic groups of these polymers and the colloidal particles [16]. It was reported that the EDCs were negatively charged and had a hydrophobic characteristic, and thereby it stably existed in the aquatic environment in a way of the negatively charged colloidal particles $[9,17]$. Therefore, in this study, the ternary copolymer P(AM-DAC- AODBAC) (PADA) was proposed to be prepared through the ultraviolet- (UV-) initiated copolymerization method using AM, DAC, and AODBAC as monomers, and the as-prepared PADA was used to remove the estrone (E1) that was a common and representative member of EDCs. In addition, the flocculation performance and mechanism should be deeply studied and researched to ignite fire in the EEs removal by flocculants.

In view of all the aforementioned aspects, this study aimed to synthesize the hydrophobic ternary copolymer PADA for the E1 removal through UV-initiated copolymerization method using AM, DAC, and AODBAC as monomers. The FT-IR spectroscopy and ${ }^{1} \mathrm{H}$ NMR spectroscopy were employed to characterize the structural characters of the prepared copolymer PADA. Besides, the morphological and thermal decomposition properties of the PADA could be obtained by the SEM and TG/DSC analyses. Then, the flocculation performance of PADA was evaluated by the E1 removal, and the optimal flocculation conditions were systematically investigated. Finally, the possible mechanism involved in the flocculation process was studied and discussed.

\section{Materials and Methods}

2.1. Materials. The industrial grade monomers acrylamide (AM), acryloyloxyethyl dimethylbenzyl ammonium chloride (AODBAC) ( $80 \mathrm{wt} \%$ in water), and acryloyloxyethyltrimethyl ammonium chloride (DAC) were obtained from Chongqing Lanjie Tap Water Company (Chongqing, China), Shanghai Orgchem Chemical Co., Ltd. (Shanghai, China), and Guangchuangjing Chemical Industry Co., Ltd. (Shanghai, China), respectively. The photo initiator $2,2^{\prime}$-azobis [2(2-imidazolin-2-yl) propane] dihydrochloride (VA-044) was purchased from Shanghai Nuohey Chemical Technology Co., Ltd. (Shanghai, China) and it was of analytical grade. The above reagents were used in the experiment without further purification. The water used in the experiment was deionized water and the E1 was purchased from Wuhan Jinglong Chemical Co. Ltd. (Wuhan, China). The copolymer of AM and DAC (PAD) was chosen for the comparable analysis.

2.2. Preparation of Copolymers. UV-initiated copolymerization was used to prepare PADA. Firstly, predetermined amounts of AM, DAC, AODBAC, VA-044, and deionized water were first added to a $100 \mathrm{~mL}$ reaction vessel and the molar ratio of DAC to AODBAC kept 3:1. Then the $\mathrm{pH}$ of the solution was adjusted to 5 with $0.1 \mathrm{~mol} \mathrm{~L}^{-1} \mathrm{HCl}$ or $\mathrm{NaOH}$, followed by a 30 min nitrogen gas bubbling at room temperature. At last, the reaction vessel was sealed and exposed to the UV radiation (wavelength, $365 \mathrm{~nm}, 200 \mathrm{~W}$, Shanghai Jiguang Special Lighting Electric Factory Co., Ltd., China) for 30-45 min at room temperature. After that, the product was purified by alcohol and acetone and dried in a vacuum oven at $60^{\circ} \mathrm{C}$ for 24 hours. Meanwhile, the copolymer PAD was prepared in a similar way as that of PADA. The possible reaction scheme of PADA was shown in Figure 1.

2.3. Characterization of Copolymers. The intrinsic viscosities and the cationic degree (CD) of polymers were determined by using an Ubbelohde viscosity meter (Shanghai Shenyi Glass Instrumental Co. Ltd., China) at $30^{\circ} \mathrm{C}$ and colloid titration method, respectively $[15,18]$. In addition, the FT-IR spectra of polymers were conducted on a 550 Series II infrared spectrometer (Mettler Toledo Instruments Co., Ltd., Switzerland) using $\mathrm{KBr}$ pellets. The ${ }^{1} \mathrm{H}$ NMR spectra of polymers were performed on an Avance 500 nuclear magnetic resonance spectrometer (Bruker Company, Ettlingen, Germany) using $\mathrm{D}_{2} \mathrm{O}$ as solvent. The SEM of the polymers was examined by the MIRA 3 LMU SEM system (TES-CAN Company, Czech Republic). Thermogravimetric (TGA) and differential scanning calorimetry (DSC) were recorded with a DTG-60H synchronal thermal analyzer (Shimadzu, Kyoto, Japan) to investigate the thermostabilities of the polymers at a heating 


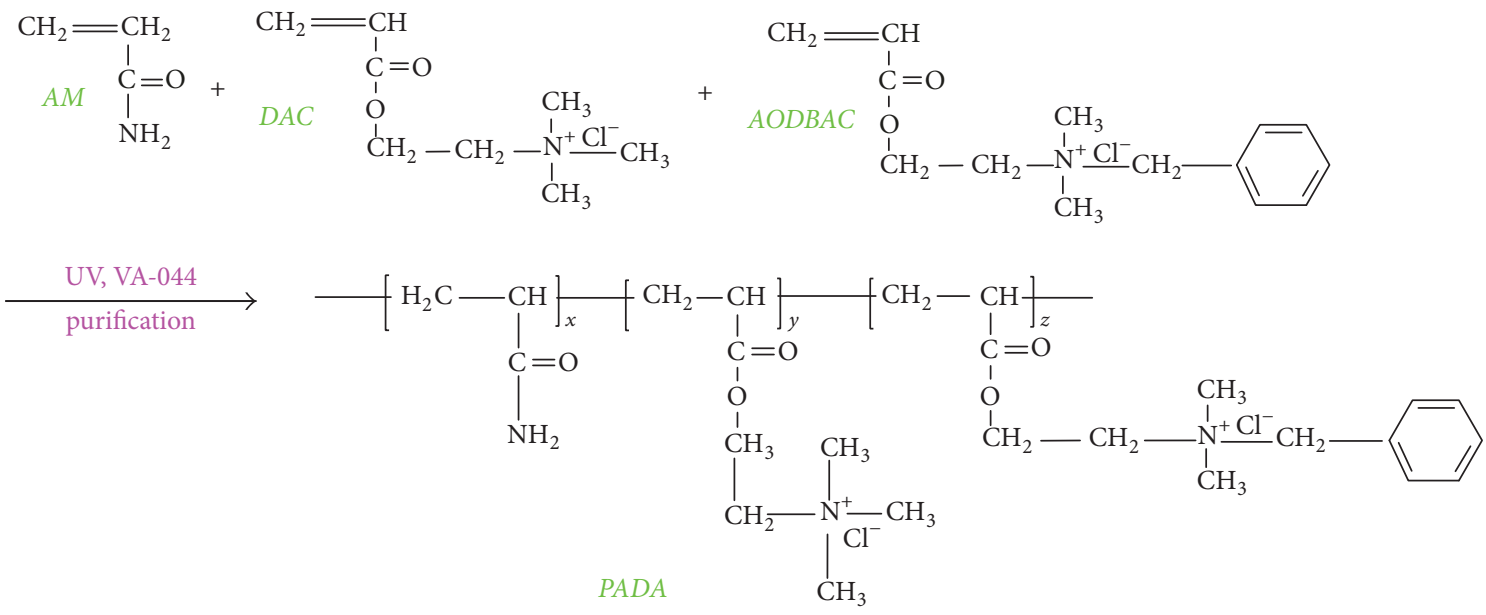

FIgURE 1: The possible reaction scheme for synthesis of PADA.

TABLE 1: The details of flocculants used in this study.

\begin{tabular}{lccc}
\hline Flocculants $^{\mathrm{a}}$ & $\begin{array}{c}\text { Intrinsic viscosity } \\
\left(\mathrm{dL} \cdot \mathrm{g}^{-1}\right)\end{array}$ & $\begin{array}{c}\text { Cationic } \\
\text { degree }(\%)\end{array}$ & $\begin{array}{c}\text { Conversion } \\
\text { rate }(\%)\end{array}$ \\
\hline PAD & 4.04 & 30.0 & 99.7 \\
PADA-1 & 4.04 & 30.0 & 99.6 \\
PADA-2 & 6.30 & 30.0 & 99.8 \\
PADA-3 & 6.30 & 40.0 & 99.7 \\
\hline
\end{tabular}

${ }^{a}$ PADA- $X(X=1,2$, and 3): copolymer of AM, AODBAC, and DAC by UVinitiated copolymerization and PAD was the copolymer of AM and DAC by UV-initiated copolymerization.

rate of $10^{\circ} \mathrm{C} \cdot \mathrm{min}^{-1}$ and a nitrogen flow of $20 \mathrm{~mL} \cdot \mathrm{min}^{-1}$ from 20 to $600^{\circ} \mathrm{C}$. The details of the polymers used in this study were shown in Table 1.

2.4. Analytical Methods and Jar Testing. The HPLC (Shimadzu, Japan) equipped with a COSMOSIL 3C18-MS-II column $(5 \mu \mathrm{m}, 4.6 \times 150 \mathrm{~mm}$, Nacalai Tesque, Inc., Japan $)$ was used to determine the content of the El. Specifically, an aliquot of solution sample (about $2 \mathrm{~mL}$ ) was filtered through $1 \mu \mathrm{m}$ glass fiber at various time interval before analyzing by HPLC. Besides, aliquots of $10.0 \mathrm{~mL}$ solution sample were injected to the HPLC running with a mobile phase of a mixture of water/acetonitrile $(v / v=55: 45)$ at the flow rate of $1.0 \mathrm{~mL} \mathrm{~min}^{-1}$, and the detected wavelength was set at $\lambda$ $=205 \mathrm{~nm}$ and the calibration curve for E1 measurement was shown in Figure 2. The flocculation experiments were conducted on a program-controlled Jar-test apparatus (ZR46, Zhongrun Water Industry Technology Development Co. Ltd., China) at ambient temperature and the initial E1 concentration was determined as $750 \mu \mathrm{g} / \mathrm{L}$. The flocculation test contained a rapid mixing ( $200 \mathrm{rpm} 1 \mathrm{~min}$ ), a slow mixing (50 rpm, $15 \mathrm{~min})$, and a settling $(10 \mathrm{~min})$ and the size of the formed E1 floc was measured using a laser diffraction instrument (Mastersizer 2000, Malvern, UK). Meanwhile, a Zetasizer Nano ZS90 (Malvern Instruments Ltd., Malvern, UK) was used to record the zeta potential of the supernatant. The method for the E1 removal rate was described in

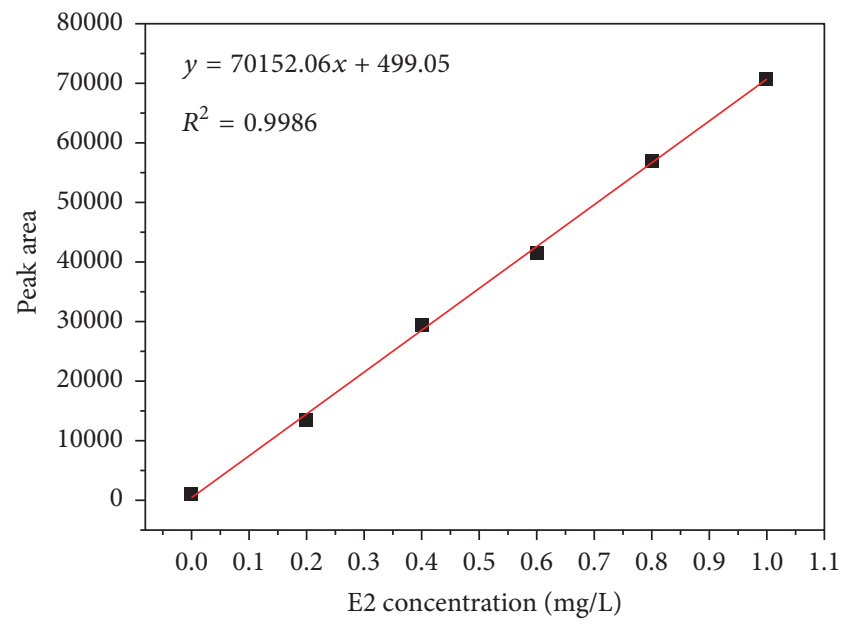

FIgURE 2: The calibration curve for E1.

Section 4.3. The final result was obtained from the average of three duplicate tests.

\section{Results and Discussion}

\subsection{Characterization of Flocculants}

3.1.1. FT-IR Spectral Analysis. As shown in Figure 3, the FTIR spectra of PAD and PADA were investigated. The PAD and PADA displayed many similar adsorption peaks as follows. The stretching vibrations of $-\mathrm{NH}_{2}$ and $\mathrm{C}=\mathrm{O}$ groups in $\mathrm{AM}$ were at 3441 and $1667 \mathrm{~cm}^{-1}$, respectively [15]. The asymmetric stretching vibrations of $-\mathrm{CH}_{3}$ and $-\mathrm{CH}_{2}-$ groups were at 2941 and $2844 \mathrm{~cm}^{-1}$, respectively. The bending vibration of $-\mathrm{CH}_{2}-$ in $-\mathrm{CH}_{2}-\mathrm{N}^{+}$group of DAC was at $1453 \mathrm{~cm}^{-1}$ [19]. The bending vibration of $-\mathrm{N}^{+}-\left(\mathrm{CH}_{3}\right)_{3}$ in DAC was observed at $961 \mathrm{~cm}^{-1}$ [20]. Compared with PAD, the characteristic adsorption peaks of PADA were observed at 774 and $707 \mathrm{~cm}^{-1}$, which were assigned to the benzene skeleton vibration in AODBAC [21]. Based on the above FT-IR spectra analysis, it was indicated that the ternary copolymer PADA 


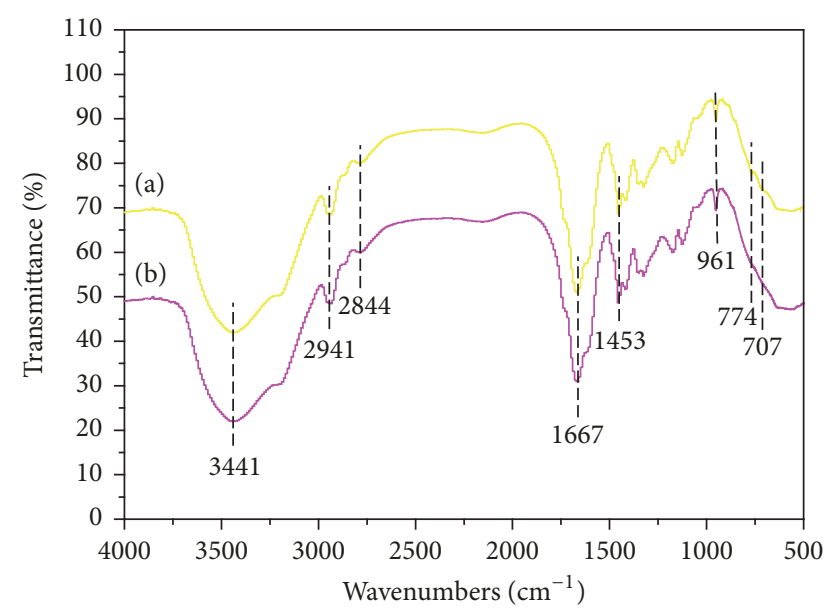

Figure 3: FT-IR spectra of (a) PADA and (b) PAD.

was successfully copolymerized by AM, DAC, and AODBAC through the UV-initiated copolymerization.

3.1.2. ${ }^{1} H$ NMR Spectral Analysis. Parallel to the FT-IR spectra, the ${ }^{1} \mathrm{H}$ NMR spectra of PAD and PADA were investigated in Figure 4 to further analyze their structure properties. PAD and PADA showed the following similar resonance peaks. The resonance peaks for the protons of $-\mathrm{CH}_{2}-$ (a) and $-\mathrm{CH}-$ (b) at backbone of the polymer were observed at $1.63 \mathrm{ppm}$ and $2.19 \mathrm{ppm}$, respectively [22]. The resonance peaks for the protons of $-\mathrm{O}-\mathrm{CH}_{2}-$ (c) were observed at $4.61 \mathrm{ppm}$. The resonance peaks for the protons of $-\mathrm{CH}_{2}-\mathrm{N}^{+}(\mathrm{d})$ group were illustrated at $\delta=3.77 \mathrm{ppm}$ [20]. Besides, the sharp resonance peak at $\delta=4.80 \mathrm{ppm}$ was associated with the protons of solvent $\mathrm{D}_{2} \mathrm{O}$. The resonance peaks for the protons of the $-\mathrm{CH}_{3}$ (e) bonded with the quaternary ammonium $\mathrm{N}^{+}$were observed at $3.21 \mathrm{ppm}$ for PAD and $3.30 \mathrm{ppm}$ for PADA. The difference was caused by the chemical environment change of the PADA, and thus slight peaks of resonance peaks occurred for the protons of the $-\mathrm{CH}_{3}(\mathrm{e})$. Compared with $\mathrm{PAD}$, the characteristic resonance peaks of PADA observed at $\delta=$ $4.55 \mathrm{ppm}(\mathrm{f})$ and $\delta=7.54 \mathrm{ppm}(\mathrm{g})$ were assigned to protons in the $-\mathrm{CH}_{2}-$ group connected to the phenyl group and protons of the phenyl group in AODBAC, respectively [21]. The corresponding resonance peaks of AM, DAC, and AODBAC were all observed, thereby indicating that the copolymerization reaction of AM, DAC, and AODBAC successfully occurred and PADA was successfully synthesized.

3.1.3. SEM of Polymers. The SEM of the polymers was investigated and the results were displayed in Figure 5. The surface morphologies of PAD and PADA were visually different. In Figure 5(a), the surface of PAD was rough and it showed a sheet-like morphology. However, considerable changes had occurred in the surface morphology of PADA when AODBAC monomer was grafted on PADA. The introduction of AODBAC greatly improved the pore size and the irregularity of the PADA surface. The originally ordered structure of PAD was destroyed by the grafting of hydrophobic AODBAC, thus leading to a porous structure with a prodigious surface area which was favorable for the improving of flocculation and water solubility ability of PADA. Besides, Figure 5(b) also showed a linear correlation of the natural logarithms of the perimeter $(L)$ and the area $(A)$, indicating that the fractal dimension of the polymer surface could be calculated using Image-Pro Plus 6.0 software [22,23]. The results showed that the fractal dimensions of PAD and PADA were 1.236 and 1.403 , respectively. This difference in morphological structure between the polymers indicated that PADA had a much higher jumbled and irregular surface morphology than that of PAD and was beneficial for the flocculation improvement [9].

3.1.4. DSC/TGA Analysis. To assess the thermal stability of the copolymer PADA, the DSC/TGA analysis was used. There were three obvious weight loss stages in the thermal weight curve of PADA in Figure 6. The first stage (30-200 ${ }^{\circ} \mathrm{C}$, weight loss of $15.4 \mathrm{wt} \%$ ) was ascribed to the moisture evaporation of adsorbed and bound water [23]. The second stage $\left(200-350^{\circ} \mathrm{C}\right.$, weight loss of $\left.20.1 \mathrm{wt} \%\right)$ was attributed to the imine reaction of the amide group as well as the thermal decomposition of methyl in the quaternary ammonium groups [24]. The third stage of mass loss occurred in the ranges of $350-550^{\circ} \mathrm{C}$ for PADA with a mass loss of about $49.8 \%$, which resulted from thermal decomposition of the copolymer backbone. Based on the above DSC/TGA analytical results, it indicated that PADA had a favorable thermal stability. Meanwhile, the DSC/TGA analysis could also verify the successful synthesis of PADA from another point of view.

\section{Flocculation Performance}

4.1. Effect of Flocculant Dosage on E1 Removal. As shown in Figure 7, the effect of flocculant dosage on E1 removal was examined. With the increased dosage of the flocculants (PADA-3, PADA-2, PADA-1, and PAD) from $1 \mathrm{mg} / \mathrm{L}$ to $4 \mathrm{mg} / \mathrm{L}$, the E1 removal all increased to the maximum, respectively. However, the E1 removal was observed to decline when the dosage of the flocculants further increased $(>4 \mathrm{mg} / \mathrm{L})$. Positively charged cationic flocculants interacted with E1 and produced electrostatic attraction, leading to the formation of small E1 flocs and the increasing of E1 removal rate. Nevertheless, the excessive dosage of cationic flocculant would strengthen the electrostatic repulsion force, thus resulting in a decrease of the E1 removal rate. Meanwhile, it was illustrated that the PADA-3 with the highest cationic degree and intrinsic viscosity had the most excellent flocculation performance among the flocculants and the order is as follows: PADA-3 (E1 removal rate: $90.1 \%$ ) > PADA-2 (E1 removal rate: $76.8 \%$ ) > PADA-1 (E1 removal rate: 65.7\%). The above phenomenon could be explained as follows: the high cationic degree and intrinsic viscosity would result in a high enhancement of bridging and charge neutralization ability, and thereby it favored a better flocculation performance. In addition, the comparison made between PADA-1 and PAD suggested that PADA-1 with hydrophobic groups (phenyl group) had a higher E1 removal efficiency. As previously researched, the hydrophobic cationic flocculant PADA-1 could improve the 


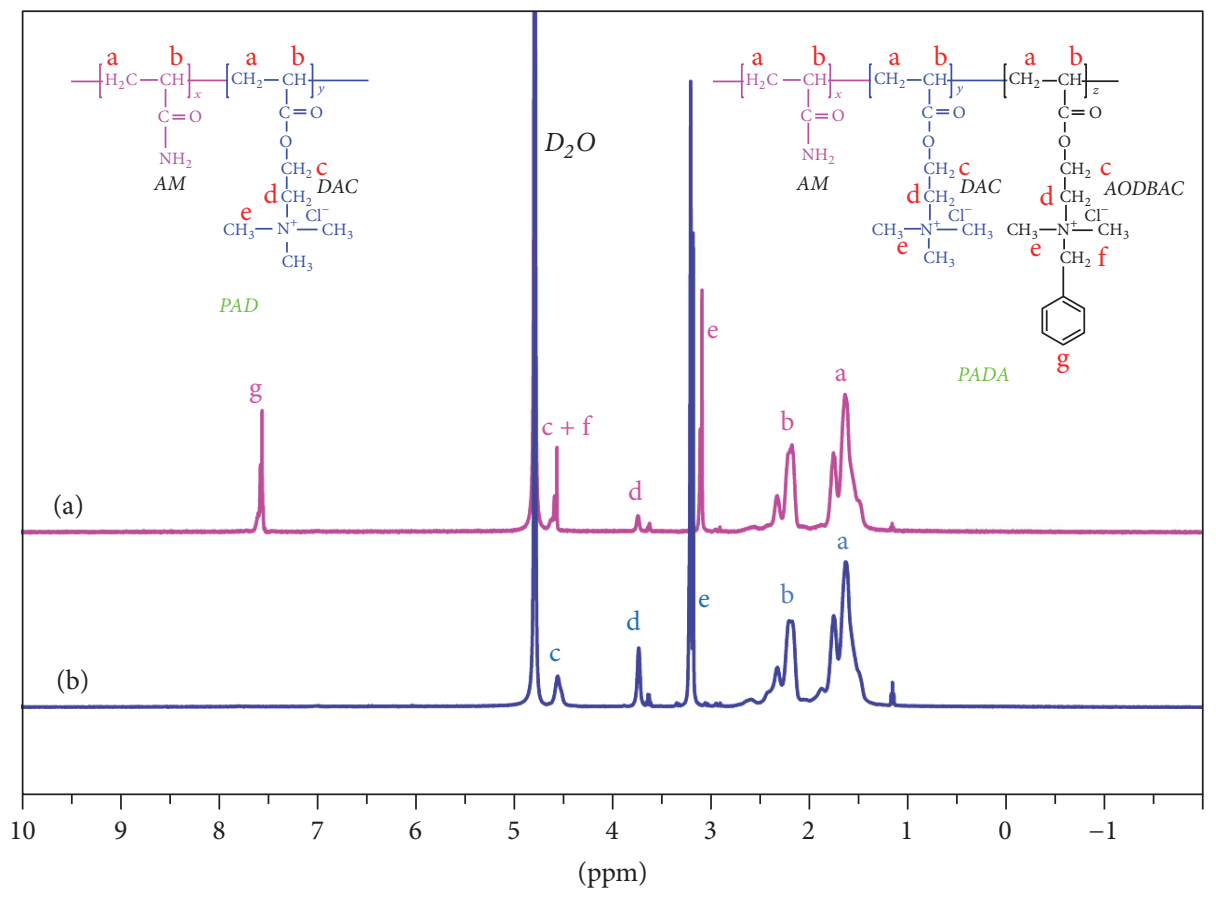

Figure $4:{ }^{1} \mathrm{H}$ NMR spectrum of (a) PADA and (b) PAD.

capacity of adsorption and bridging, as well as reinforcing the interaction between E1 particles and the flocculant [25].

4.2. The Effect of $\mathrm{pH}$ on E1 Removal. The $\mathrm{pH}$ played a crucial role in the process of E1 flocculation and removal, and the acid or alkali condition had evident effects on the surface property of the flocculants [26]. Meanwhile, it was also reported that the acid or alkali condition also affected the flocculation mechanism (charge neutralization and bridging) [9]. Therefore, the effect of the $\mathrm{pH}$ on the $\mathrm{E} 1$ removal was investigated at the flocculant dosage $(4 \mathrm{mg} / \mathrm{L})$ and the results were shown in Figure 8. With the increased $\mathrm{pH}$ from 1 to 7 , the E1 removal rate of the flocculants all significantly increased and reached the top value. However, with the continually increased $\mathrm{pH}$ from 7 to 11, low efficiencies of the flocculants on the E1 removal rate were observed. The maximum value of E1 removal rate for the four flocculants (PADA-3: 90.1\%, PADA-2: 76.8\%, PADA-1: 65.7\%, and PAD: 57.1\%) was all obtained at $\mathrm{pH}=7$, which indicated that the neutral condition $(\mathrm{pH}=7)$ was more favorable for the E1 removal. The relatively strong acid environment could change the surface property of the flocculants and caused the protonation effect of amino group $\left(-\mathrm{NH}_{3}{ }^{+}\right)$and the cross-linking effect of polymer chain, thus worsening the liner extension of the polymer and weakening the polymer bridging ability [27]. Besides, the excessive $\mathrm{H}^{+}$ions associating with the strong acid environment resulted in the strong electrostatic repulsion between the E1 colloids and prevented the floc growth and decreased the flocculation efficiency. Conversely, the strong alkaline condition was detrimental for the E1 removal and the flocculants performed undesirably at this condition. On one hand, the $-\mathrm{NH}_{2}$ group of the flocculants tended to hydrolyze under the condition of a large numbers of negatively charged
$\mathrm{OH}^{-}$groups. On the other hand, the E1 colloids were wrapped by amount of $\mathrm{OH}^{-}$groups to form a negative group. As a result, the electrostatic repulsion between the E1 colloids increased and weakened the flocculants charge neutralization ability, which caused a declination of E1 removal by the flocculant. The neutral condition generated the advantages of a facile extension of the polymers chain and a low charge repulsion, and thereby an excellent flocculation performance of the E1 removal was observed. In Figure 7, it was obvious that the PADA-3 had the best flocculation performance on the E1 removal among the flocculants, which indicated that PADA-3 with relatively high cationic degree and molecular weight was favorable for the E1 removal. Finally, it was worth noting that PADA-1 performed efficiently on the E1 removal than that of PAD. This phenomenon could be explained by the following reasons. The PADA-1 hydrophobically modified by introducing a hydrophobic monomer (phenyl group) had a special rheological characteristics of improving the liner spreading of the PADA-1 chain and enhancing the solution viscosity, thus forming more active points to capture E1 colloidal particles to form large flocs through bridging effect. In addition, the grafting of phenyl group on the PADA1 polymer chain could lead to a porous structure with a prodigious surface area illustrated in Section 3.1.3, which was beneficial for the improving of flocculation adsorption ability and water solubility ability of PADA-1 than that of PAD. As a result, the flocculation performance of PADA-1 on the E1 removal was extremely enhanced, compared with that of PAD. Meanwhile, more than 50\% E1 removal rate could be obtained by using the modified ternary copolymer PADA3 on the $\mathrm{pH}$ range of $4-11$, which indicated that the PADA3 had a wide application in the process of practical EDCs contaminative water treatment. 

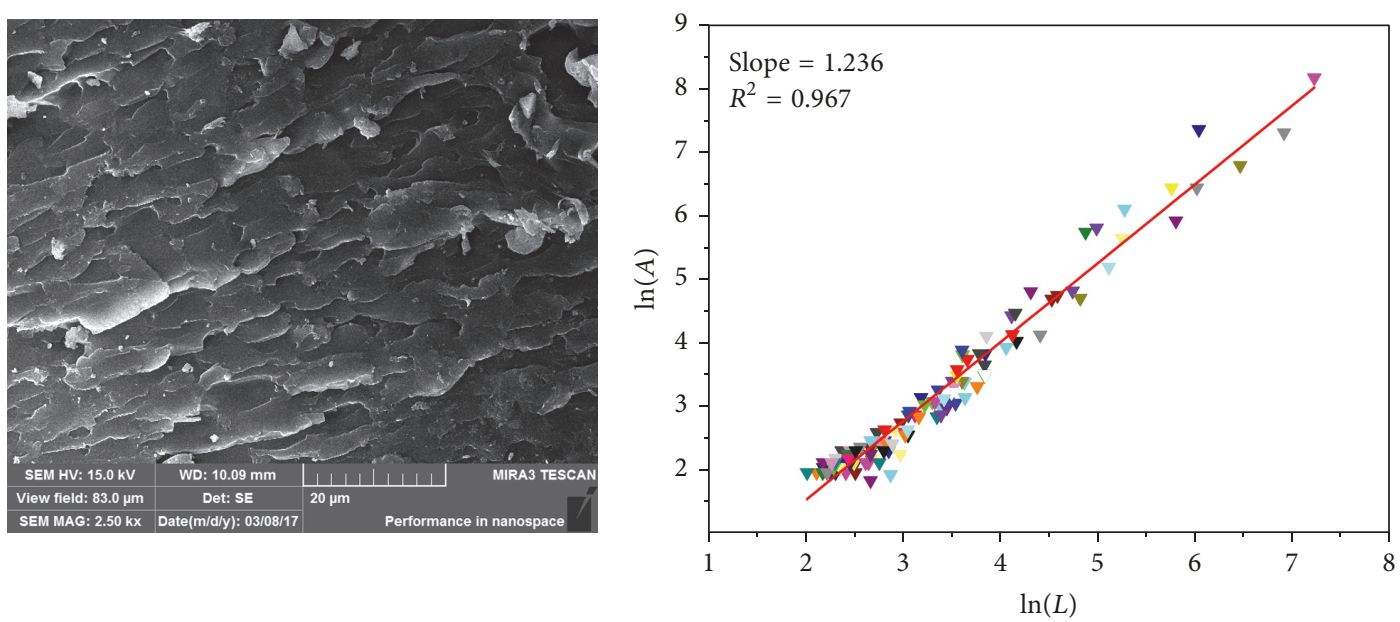

(a)
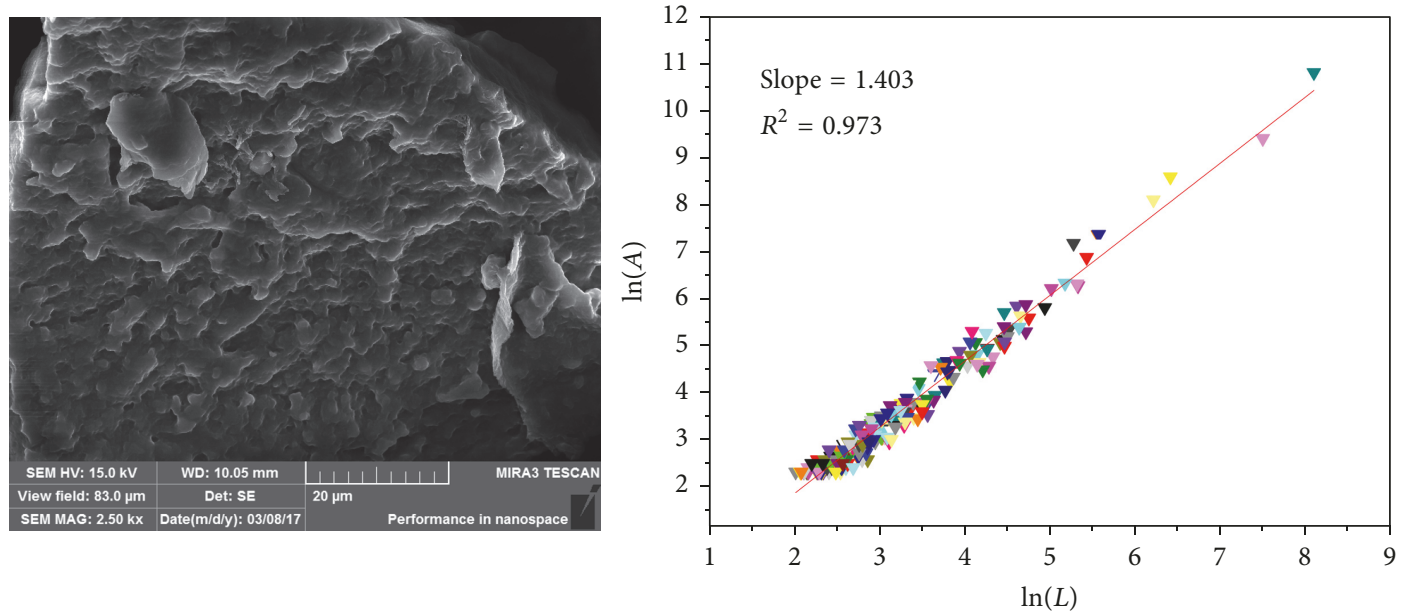

(b)

Figure 5: SEM images of (a) PAD and (b) PADA.

4.3. Kinetic Investigation. To investigate the effect of the flocculants on the E1 removal, the flocculation kinetics analysis was performed at the optimal conditions (dosage $=$ $\left.4 \mathrm{mg} \cdot \mathrm{L}^{-1}, \mathrm{pH}=7\right)$. The E1 concentration for each flocculant was recorded from the termination of the rapid mixing and it lasted $21 \mathrm{~min}$ with a time interval of $3 \mathrm{~min}$. Based on the recording results of the E1 concentration, the E1 removal rate for each flocculant was calculated by (1) and the result of the E1 removal rate was displayed in Figure 9(a).

$$
\text { E1 removal rate }=\frac{N_{t}}{N_{0}}(\%),
$$

where $N_{t}$ was the E1 concentration at different flocculation time of $3,6,9,12,15,18$, and $21 \mathrm{~min}$ and $N_{0}$ was the initial E1 concentration $(750 \mu \mathrm{g} / \mathrm{L})$.

The kinetic calculation method was expressed in (2) according to the previous literature [28].

$$
\left(\frac{N_{0}}{N_{t}}\right)^{1 / 2}=1+0.5 \times k N_{0},
$$

TABLE 2: The flocculation kinetics $\left(k N_{0}\right)$ for the four flocculants (PADA-3, PADA-2, PADA-1, and PAD).

\begin{tabular}{lcc}
\hline Flocculants & Flocculation kinetics $k N_{0}\left(\times 10^{-4} \mathrm{~s}^{-1}\right)$ & $R^{2}$ \\
\hline PADA-3 & 22.69 & 0.953 \\
PADA-2 & 10.95 & 0.959 \\
PADA-1 & 9.77 & 0.972 \\
PAD & 4.81 & 0.981 \\
\hline
\end{tabular}

where $N_{0}$ and $N_{t}$ were the same as those expressed in (1) and $k$ was the kinetic constant in the flocculation system. The flocculation kinetics $k N_{0}$ value was presented in Table 2.

As shown in Figure 9(a), the variation of the E1 removal rate for the four flocculants increased rapidly from the first 9 min and then slightly increased to a top value. It was evident that the curve for the PADA-3 increased sharply during the first of $9 \mathrm{~min}$ among the flocculants. Meanwhile, the slope $\left(k N_{0}\right)$ of the simulated curve for the four flocculants was generally in the following order: PADA-3 > PADA-2 > PADA-1 > PAD. The slope value for the PADA-3 $\left(k N_{0}=\right.$ 


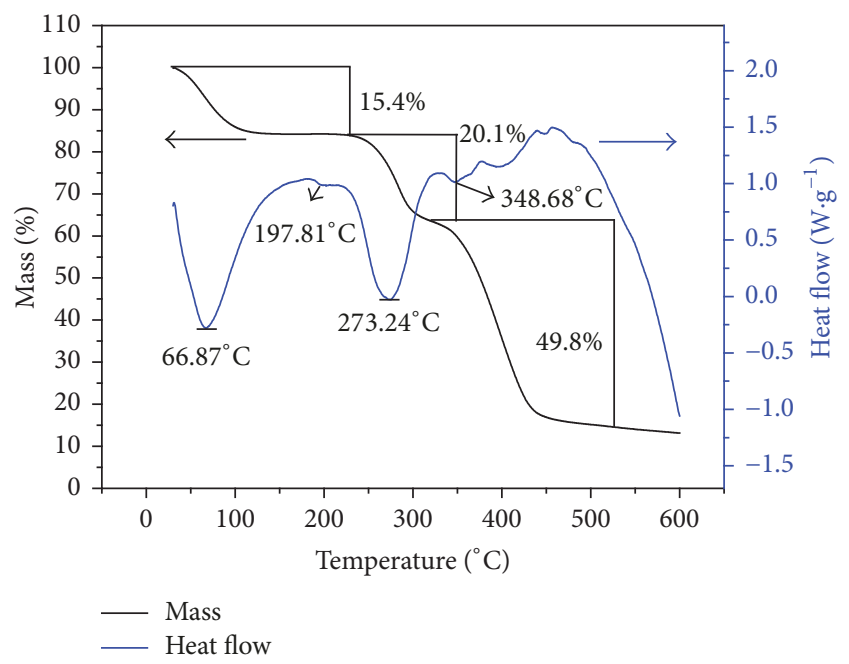

Figure 6: Thermal gravimetric curve of PADA.

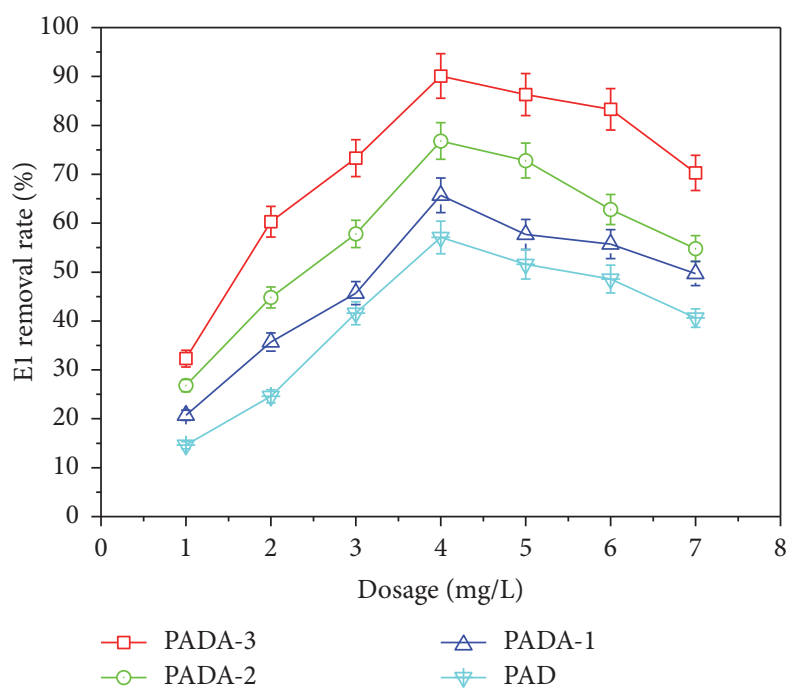

Figure 7: The effect of flocculant dosage on the E1 removal.

$22.69 \times 10^{-4} \mathrm{~s}^{-1}$ ) reached the largest among the flocculants, which indicated that the relatively high cationic degree and intrinsic viscosity with PADA-3 resulted in a prominent E1 flocculation performance, and the E1 removal efficiency was greatly improved. On one hand, the strong charge neutralization ability with the high cationic degree could attract and neutralize the negatively charged E1 colloids completely, thus causing E1 colloids to lose their stabilization. On the other hand, the polymer chain of the flocculant with the high intrinsic viscosity would efficiently intercept the E1 colloids and attach on the surface of the E1 colloids, and the remaining part of the polymer chain would continue to capture the approaching E1 colloids to form large floc structure. In this process, the charge neutralization and bridging mechanism jointly controlled the colloidal process and they played a key role in producing larger flocs. Besides, the preparation made between the PADA-1 and PAD suggested that the introducing of phenyl group on the PADA-1 could significantly improve

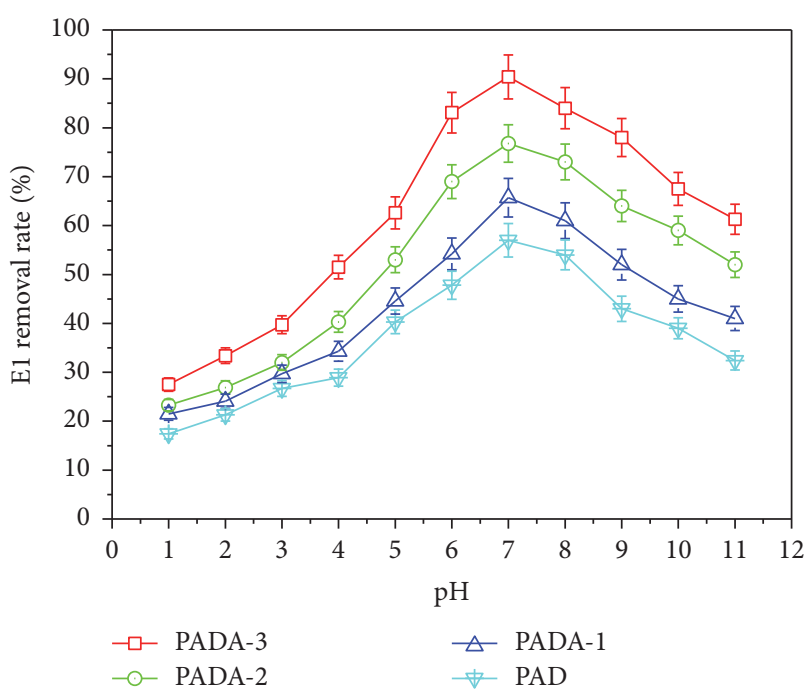

Figure 8: The E1 removal rate after flocculation at various $\mathrm{pH}$.

the flocculation performance at the same cationic degree and intrinsic viscosity. An explanation could be made as that the hydrophobic phenyl group in the PADA-1 polymer chain caused the polymer chain to extend well and more efficient molecular collisions took place between PADA-1 polymer chain and suspended E1 colloidal particles. As a result, more active sites of the polymer chain were exposed to improve the bridging effect.

4.4. Effect of the E1 Concentration. In real water samples, the concentration of EDCs varied greatly lying on the sample to which it applied. As such, the flocculation performance correspondingly changed. Therefore, different E1 concentration $(0.25,0.5,0.75$, and $1.0 \mathrm{mg} / \mathrm{L})$ was used to evaluate the impact of the E1 concentration on the PADA-3 flocculation performance on the neutral condition $(\mathrm{pH}=7)$. As shown in Figure 10, the E1 removal rate firstly increased to the maximum at the optimum dosage and then decreased with the continual increasing of dosage. At the lower dosage of flocculant, there was not enough positive charge to neutralize the negative E1 colloids and the collision between the polymer chain and E1 colloids was also insufficient to form large flocs. The desirable flocculation performance always occurred at a relatively high dosage because the flocculation process completely finished in this condition. PADA-3 as a positive charged polymer could play charge neutralization, adsorption, and bridge effects. However, when PADA-3 was overdosed, the formed flocs were turned to positive charge and restabilized, and thus it resulted in a decrease in E1 removal. Meanwhile, it was obvious that the optimal E1 concentration was $0.75 \mathrm{mg} / \mathrm{L}$ rather than $1.0 \mathrm{mg} / \mathrm{L}$. The excess E1 concentration would generate more negatively charged E1 colloids which would wrap around the formed flocs and led to a steric repulsive forces to exhibit a protection effect rather than flocculation $[29,30]$. As a result, the flocculation performance became worse. Therefore, $0.75 \mathrm{mg} / \mathrm{L}$ E1 concentration was chosen as the initial concentration to simulate actual EDCs contaminative water in this experiment. 


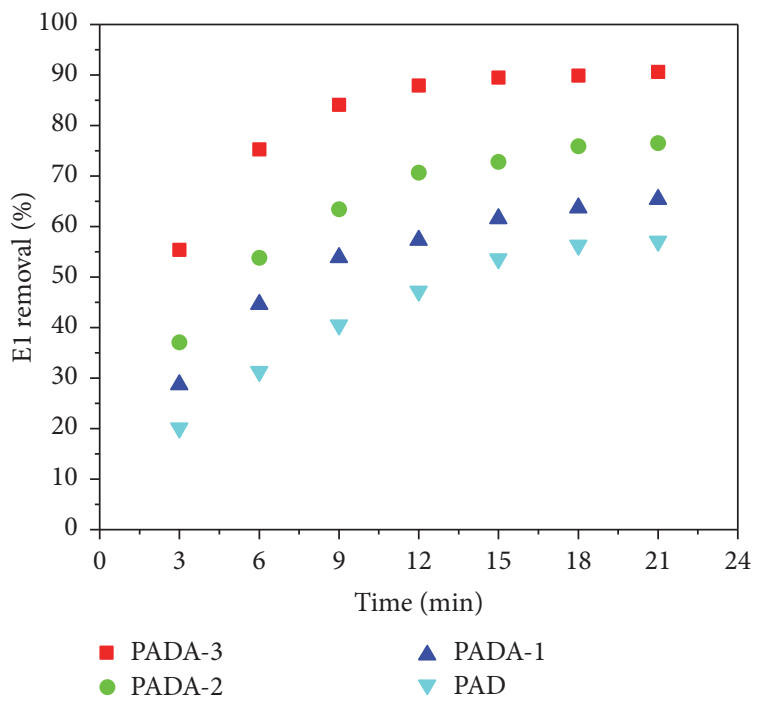

(a)

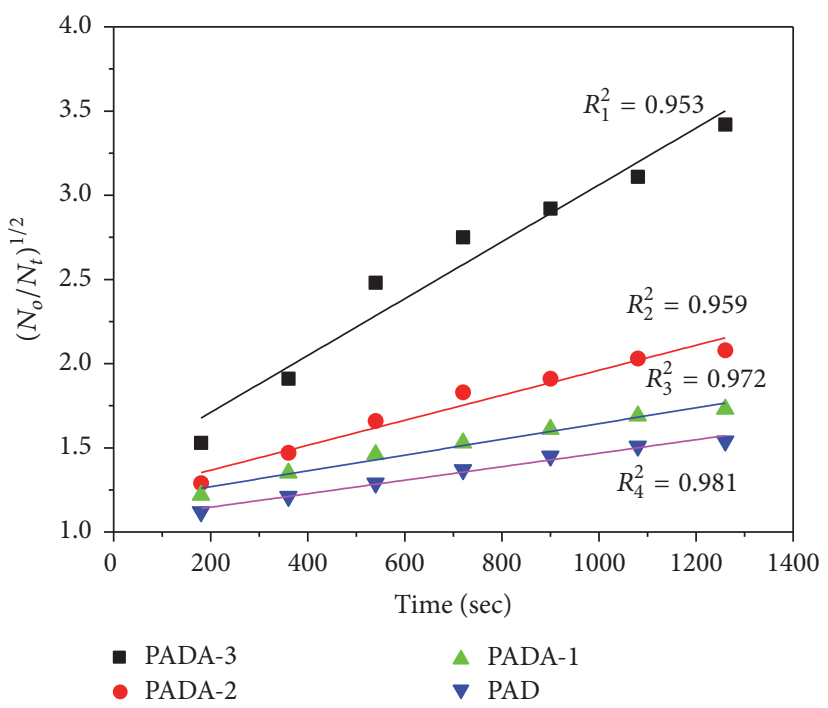

(b)

FIGURE 9: The variation of E1 removal (a) and (b) $\left(N_{0} / N_{t}\right)^{1 / 2}$ as a function of setting time for different flocculants.

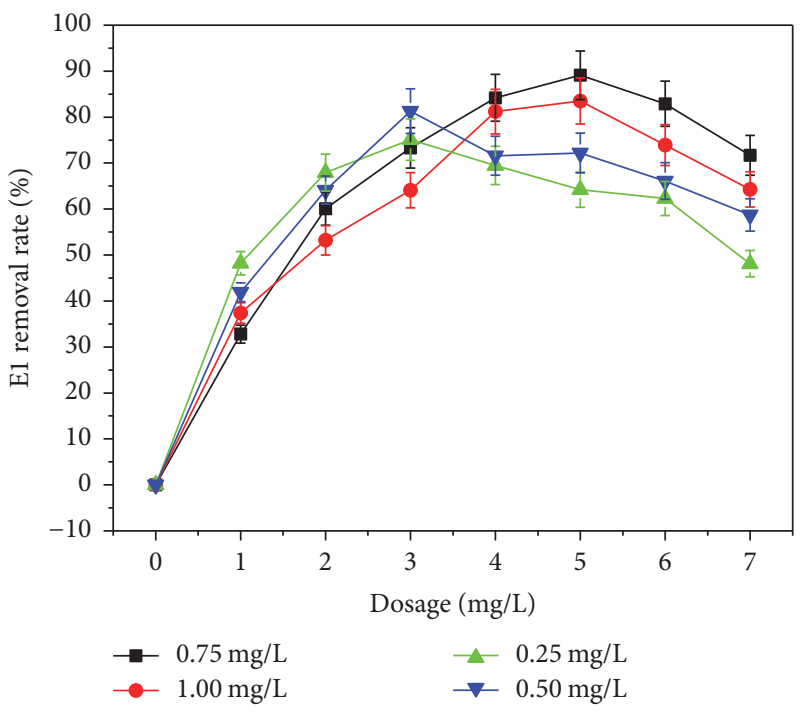

FIGURE 10: The effect of the E1 concentration on the flocculation performance (PADA-3 was used).

\section{Flocculation Mechanism}

To obtain a deep understanding of the mechanism involved in the flocculation process, the zeta potential and E1 floc size were analyzed at the $\mathrm{pH}=7$. The zeta potential investigation contributed much to the understanding of the charge characteristics of E1 colloids, and the E1 floc size was helpful to the perception of the floc aggregation properties [22].

In Figure 11(a), it revealed that the initial zeta potential of the E1 wastewater was negative and the value was $-6.9 \mathrm{mV}$ before the addition of the flocculant. In this condition, the E1 colloid was very stable in the wastewater under the electrostatic repulsion and it was difficult to be removed without any treatment. However, with the increasing dosage of the flocculants, the zeta potential of the E1 wastewater was reversed and it became positive. This phenomenon suggested that the cationic flocculant could greatly change the charge characteristics of E1 colloids and further affected the E1 flocculation performance. Compared with the PADA-2, PADA-1, and PAD, the PADA-3 with the relatively high cationic degree neutralized the negative E1 colloids more efficiently, and thus the isoelectric point was the lowest among the four flocculants, whereas the zeta potential of PADA-2, PADA-1, and PAD had no evident difference. Meanwhile, the E1 floc size of the PADA-3 $(18.3 \mu \mathrm{m})$ shown in Figure 11(b) was larger than that of PADA-2 $(16.2 \mu \mathrm{m})$. It indicated that the charge neutralization played an important role in the E1 flocculation and it could improve the E1 floc aggregation. However, in addition to charge neutralization, other flocculation mechanisms also worked in this flocculation process, which could be reflected by the following phenomenon. PADA- 2 and PADA- 1 showed the same cationic degree (30\%), whereas the E1 floc size of PADA-2 and PADA-1 was different. PADA-2 with a higher intrinsic viscosity than PADA-1 would have longer polymer chain to capture and adsorb E1 colloids to form larger E1 floc size through bridging and adsorption. Meanwhile, the optimal dosage of the flocculants was around the isoelectric point and the values were not the same; it manifested that bridging and adsorption other than charge neutralization also played an important role in the flocculation process. Besides, the floc size comparison between PADA-1 and PAD indicated that the grafting of phenyl group on the PADA-1 had a promotion effect to the bridging enhancement, and thereby PADA-1 had a larger floc size than PAD.

According to the above analyses, a possible flocculation mechanism of PADA was extracted and summarized in Figure 12. With the addition of the flocculant, more negatively charged E1 colloids were neutralized completely and destabilized, and hence the electrostatic repulsion between 


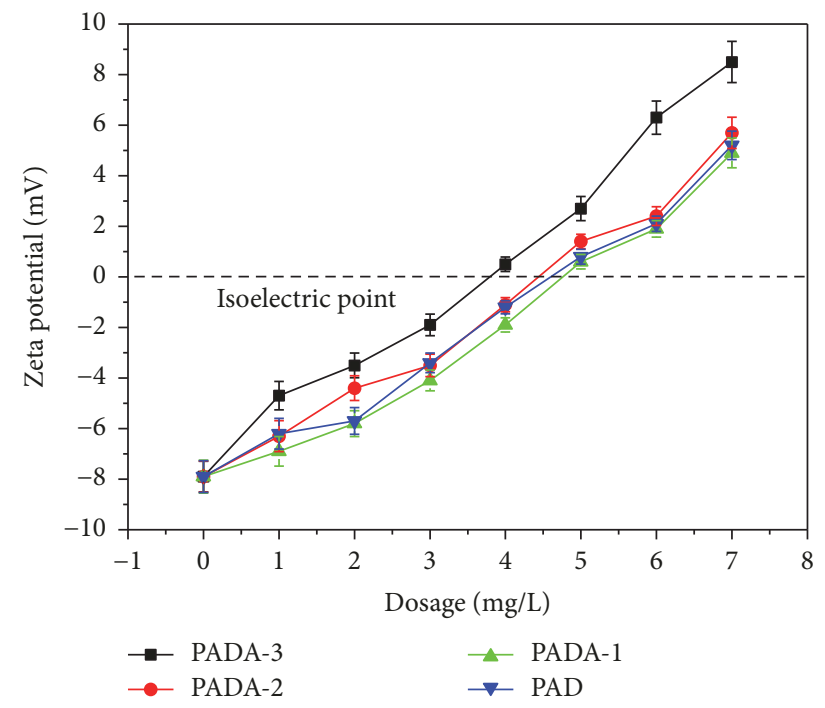

(a)

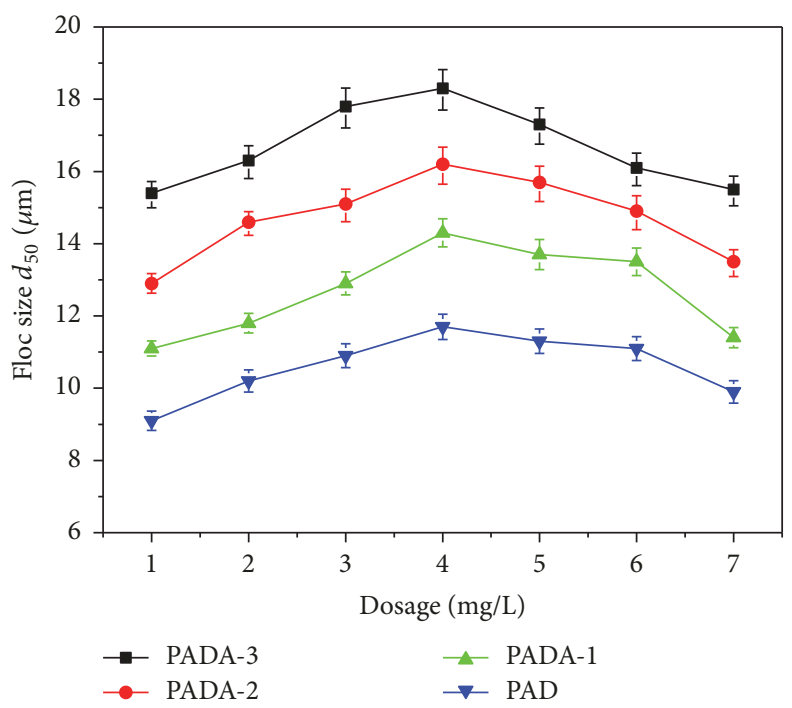

(b)

Figure 11: The zeta potential (a) and floc size (b) of the flocculants.
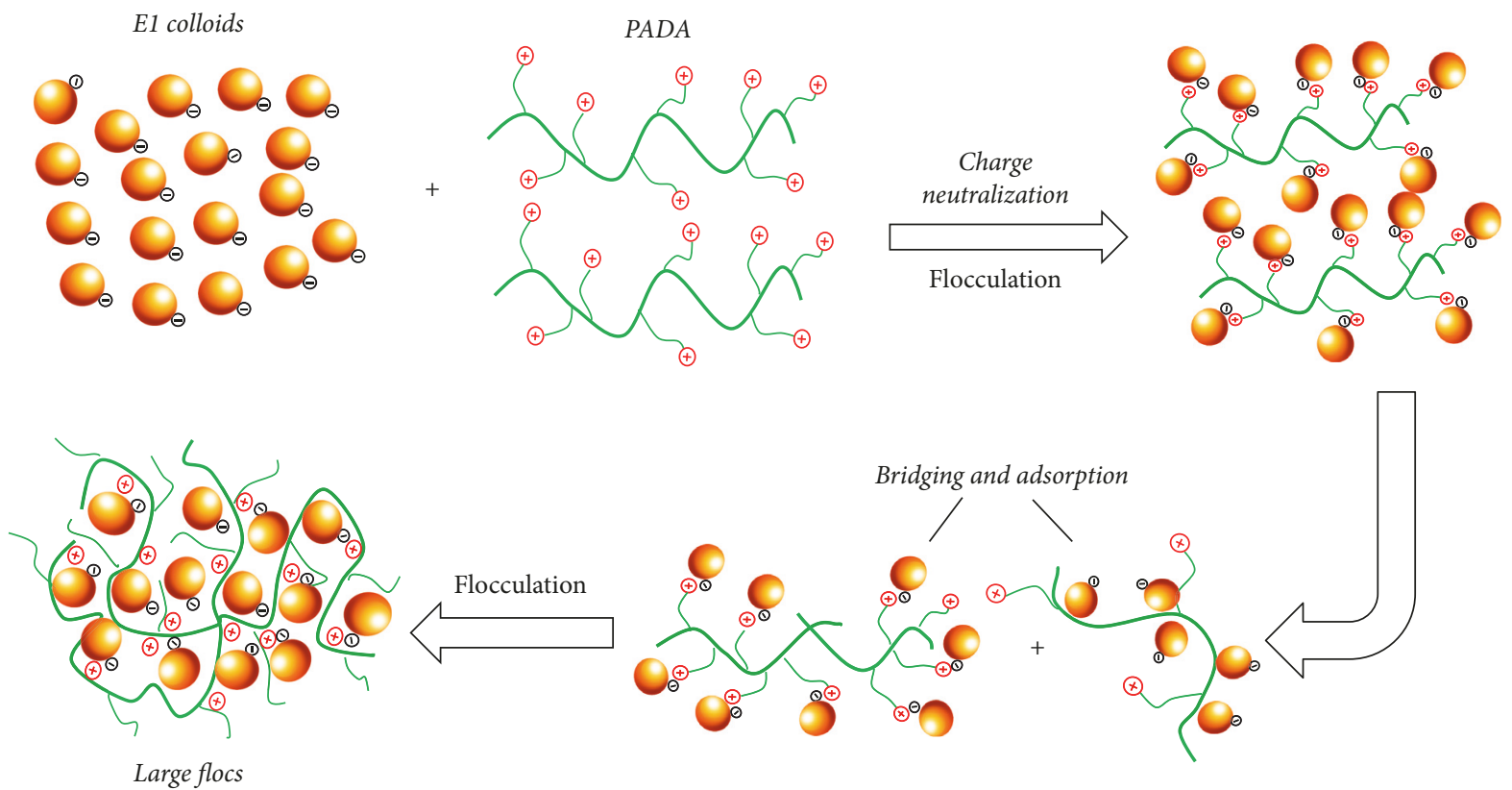

FIGURE 12: The possible flocculation mechanism of the flocculant.

the colloids reached the minimum. Subsequently, the destabilized E1 colloids were captured and adsorbed on the polymer chain under the effect of adsorption and bridging. Moreover, the hydrophobic of the property phenyl group on the PADA was helpful for the polymer chain spread to improve adsorption and bridging. Finally, the E1 colloids anchored on the polymer chain would encounter the vacant place of approaching polymer chain to aggregate and form large flocs. As a result, the E1 was removed and separated efficiently from wastewater by the flocculation method.

\section{Conclusion}

A hydrophobic flocculant PADA was successfully synthesized with AM, DAC, and AODBAC through UV-initiated copolymerization technique to remove E1 contaminative water. The main conclusions of this research were expressed as follows:

(1) The analytical results of FT-IR and ${ }^{1} \mathrm{H}$ NMR manifested the successful preparation of PADA and PAD. A favorable thermal stability of PADA was concluded by the TG/DSC analysis. Meanwhile, the SEM analysis indicated that PADA had a much higher jumbled and irregular surface 
morphology than that of PAD and could improve the E1 flocculation performance.

(2) The flocculation results showed that higher E1 removal rate was obtained by using the flocculant PADA-3 with $40 \%$ cationic degree and intrinsic viscosity of $6.30 \mathrm{dL} \cdot \mathrm{g}^{-1}$. The optimal flocculant condition was fixed at $\mathrm{pH}=7,4.0 \mathrm{mg} / \mathrm{L}$ of flocculant dosage, and $0.75 \mathrm{mg} / \mathrm{L}$ of E1 concentration. In this condition, a higher E1 removal rate of $90.1 \%$, a larger floc size of $18.3 \mu \mathrm{m}$, and a higher of flocculation kinetics of $22.69 \times 10^{-4} \mathrm{~s}^{-1}\left(k N_{0}\right)$ were obtained. Moreover, the grafting of phenyl group on the PADA could enhance the flocculation efficiency.

(3) The zeta potential and floc size analytical results revealed that the charge neutralization, adsorption, and bridging effects played a crucial role in the E1 flocculation progress. Under the synergistic effects of the above mechanism, a larger E1 floc size was formed and a higher E1 removal rate was obtained.

\section{Conflicts of Interest}

The authors declare that there are no conflicts of interest regarding the publication of this paper.

\section{Acknowledgments}

The authors are grateful for the financial support provided by the National Natural Science Foundation of China (Project nos. 51508057, 41401255, 51408082, and 51508056).

\section{References}

[1] H.-S. Chang, K.-H. Choo, B. Lee, and S.-J. Choi, “The methods of identification, analysis, and removal of endocrine disrupting compounds (EDCs) in water," Journal of Hazardous Materials, vol. 172, no. 1, pp. 1-12, 2009.

[2] A. Begum and S. K. Gautam, "Dechlorination of endocrine disrupting chemicals using $\mathrm{Mg} 0 / \mathrm{ZnCl} 2$ bimetallic system," Water Research, vol. 45, no. 7, pp. 2383-2391, 2011.

[3] C. Jung, A. Son, N. Her, K.-D. Zoh, J. Cho, and Y. Yoon, "Removal of endocrine disrupting compounds, pharmaceuticals, and personal care products in water using carbon nanotubes: A review," Journal of Industrial and Engineering Chemistry, vol. 27, pp. 1-11, 2015.

[4] Z. Qiang, H. Dong, B. Zhu, J. Qu, and Y. Nie, "A comparison of various rural wastewater treatment processes for the removal of endocrine-disrupting chemicals (EDCs)," Chemosphere, vol. 92, no. 8, pp. 986-992, 2013.

[5] L. Joseph, J. Heo, Y.-G. Park, J. R. V. Flora, and Y. Yoon, "Adsorption of bisphenol A and 17 $\alpha$-ethinyl estradiol on single walled carbon nanotubes from seawater and brackish water," Desalination, vol. 281, no. 1, pp. 68-74, 2011.

[6] D. Balabanič, D. Hermosilla, N. Merayo, A. K. Klemeničič, and Á. Blanco, "Comparison of different wastewater treatments for removal of selected endocrine-disruptors from paper mill wastewaters," Journal of Environmental Science and Health, Part A: Toxic/Hazardous Substances and Environmental Engineering, vol. 47, no. 10, pp. 1350-1363, 2012.

[7] V. Yangali-Quintanilla, A. Sadmani, M. McConville, M. Kennedy, and G. Amy, "A QSAR model for predicting rejection of emerging contaminants (pharmaceuticals, endocrine disruptors) by nanofiltration membranes," Water Research, vol. 44, no. 2, pp. 373-384, 2010.

[8] D. Błedzka, M. Gmurek, M. Gryglik, M. Olak, J. S. Miller, and S. Ledakowicz, "Photodegradation and advanced oxidation of endocrine disruptors in aqueous solutions," Catalysis Today, vol. 151, no. 1-2, pp. 125-130, 2010.

[9] H. Zheng, J. Ma, C. Zhu et al., "Synthesis of anion polyacrylamide under UV initiation and its application in removing dioctyl phthalate from water through flocculation process," Separation and Purification Technology, vol.123, pp. 35-44, 2014.

[10] F. Sher, A. Malik, and H. Liu, "Industrial polymer effluent treatment by chemical coagulation and flocculation," Journal of Environmental Chemical Engineering (JECE), vol. 1, no. 4, pp. 684-689, 2013.

[11] L. Lu, Z. Pan, N. Hao, and W. Peng, "A novel acrylamidefree flocculant and its application for sludge dewatering," Water Research, vol. 57, pp. 304-312, 2014.

[12] S. Zhao, B. Gao, Q. Yue et al., "Study of Enteromorpha polysaccharides as a new-style coagulant aid in dye wastewater treatment," Carbohydrate Polymers, vol. 103, no. 1, pp. 179-186, 2014.

[13] Z. Wu, P. Zhang, G. Zeng, M. Zhang, and J. Jiang, "Humic acid removal from water with polyaluminum coagulants: Effect of sulfate on aluminum polymerization," Journal of Environmental Engineering (United States), vol. 138, no. 3, pp. 293-298, 2012.

[14] M. Hassan, R. Abou-Zeid, E. Hassan, L. Berglund, Y. Aitomäki, and K. Oksman, "Membranes based on cellulose nanofibers and activated carbon for removal of Escherichia coli bacteria from water," Polymer, vol. 9, no. 8, article 335, 2017.

[15] L. Feng, H. Zheng, B. Gao, C. Zhao, S. Zhang, and N. Chen, "Enhancement of textile-dyeing sludge dewaterability using a novel cationic polyacrylamide: role of cationic block structures," RSC Advances, vol. 7, no. 19, pp. 11626-11635, 2017.

[16] Z. L. Yang, B. Y. Gao, C. X. Li, Q. Y. Yue, and B. Liu, "Synthesis and characterization of hydrophobically associating cationic polyacrylamide," Chemical Engineering Journal, vol. 161, no. 12, pp. 27-33, 2010.

[17] Z. Qiang, Y. Nie, W. Ben, J. Qu, and H. Zhang, "Degradation of endocrine-disrupting chemicals during activated sludge reduction by ozone," Chemosphere, vol. 91, no. 3, pp. 366-373, 2013.

[18] H. Ren, W. Chen, Y. Zheng, and Z. Luan, "Effect of hydrophobic group on flocculation properties and dewatering efficiency of cationic acrylamide copolymers," Reactive and Functional Polymers, vol. 67, no. 7, pp. 601-608, 2007.

[19] G. Zhu, J. Liu, J. Yin et al., "Functionalized polyacrylamide by xanthate for $\mathrm{Cr}$ (VI) removal from aqueous solution," Chemical Engineering Journal, vol. 288, pp. 390-398, 2016.

[20] Q. Guan, H. Zheng, J. Zhai et al., "Effect of template on structure and properties of cationic polyacrylamide: Characterization and mechanism," Industrial \& Engineering Chemistry Research, vol. 53, no. 14, pp. 5624-5635, 2014.

[21] Y. Liao, H. Zheng, L. Qian, Y. Sun, L. Dai, and W. Xue, "UV-initiated polymerization of hydrophobically associating cationic polyacrylamide modified by a surface-active monomer: A comparative study of synthesis, characterization, and sludge dewatering performance," Industrial \& Engineering Chemistry Research, vol. 53, no. 27, pp. 11193-11203, 2014.

[22] L. Feng, H. Zheng, Y. Wang, S. Zhang, and B. Xu, "Ultrasonictemplate technology inducing and regulating cationic microblocks in CPAM: characterization, mechanism and sludge 
flocculation performance," RSC Advances, vol. 7, no. 38, pp. 23444-23456, 2017.

[23] Y. Sun, M. Ren, C. Zhu et al., "UV-initiated graft copolymerization of cationic chitosan-based flocculants for treatment of zinc phosphate-contaminated wastewater," Industrial \& Engineering Chemistry Research, vol. 55, no. 38, pp. 10025-10035, 2016.

[24] J. Ma, J. Shi, H. Ding, G. Zhu, K. Fu, and X. Fu, "Synthesis of cationic polyacrylamide by low-pressure UV initiation for turbidity water flocculation," Chemical Engineering Journal, vol. 312, pp. 20-29, 2017.

[25] H. Zheng, Y. Sun, J. Guo et al., "Characterization and evaluation of dewatering properties of PADB, a highly efficient cationic flocculant," Industrial \& Engineering Chemistry Research, vol. 53, no. 7, pp. 2572-2582, 2014.

[26] J. Ma, K. Fu, X. Fu et al., "Flocculation properties and kinetic investigation of polyacrylamide with different cationic monomer content for high turbid water purification," Separation and Purification Technology, vol. 182, pp. 134-143, 2017.

[27] H. Zheng, L. Feng, B. Gao, Y. Zhou, S. Zhang, and B. Xu, "Effect of the cationic block structure on the characteristics of sludge flocs formed by charge neutralization and patching," Materials, vol. 10, no. 5, article 487, 2017.

[28] L. Feng, H. Zheng, B. Gao et al., "Fabricating an anionic polyacrylamide (APAM) with an anionic block structure for high turbidity water separation and purification," RSC Advances, vol. 7, no. 46, pp. 28918-28930, 2017.

[29] X. Huang, B. Gao, H. Rong, Q. Yue, Y. Zhang, and P. Teng, "Effect of using polydimethyldiallylammonium chloride as coagulation aid on polytitanium salt coagulation performance, floc properties and sludge reuse," Separation and Purification Technology, vol. 143, pp. 64-71, 2015.

[30] Y. Zhou, H. Zheng, B. Gao et al., "Waste activated sludge (WAS) dewatering properties of an original hydrophobically modified polyacrylamide containing a cationic microblock structure," RSC Advances, vol. 7, no. 46, pp. 28733-28745, 2017. 


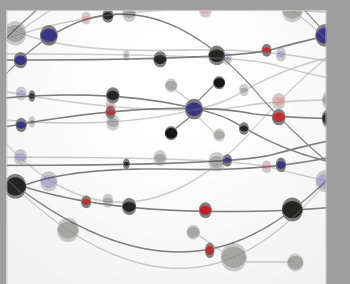

The Scientific World Journal
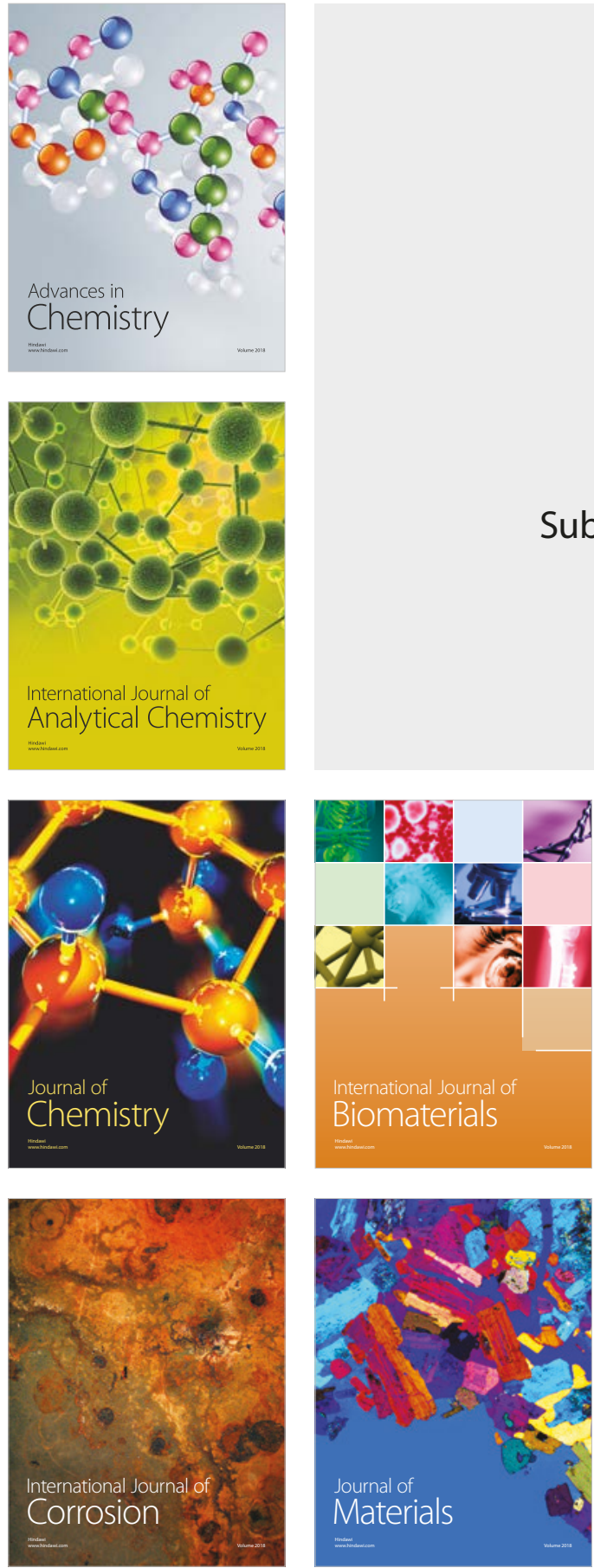

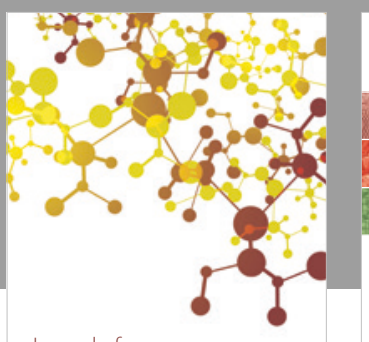

Journal of

Applied Chemistry
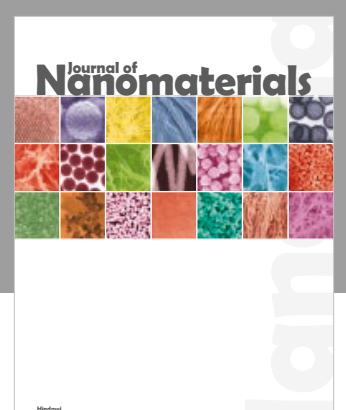

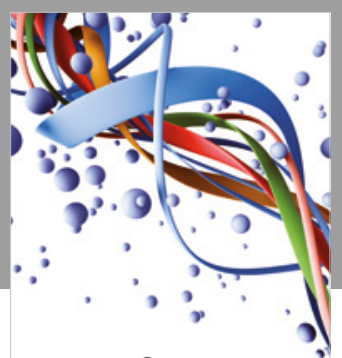

Scientifica

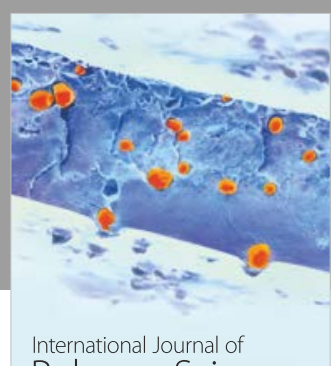

Polymer Science

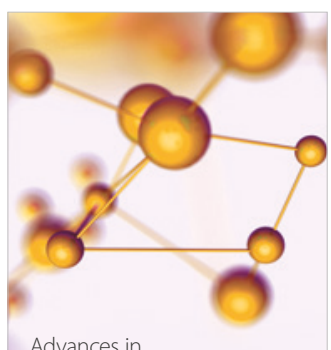

Physical Chemistry
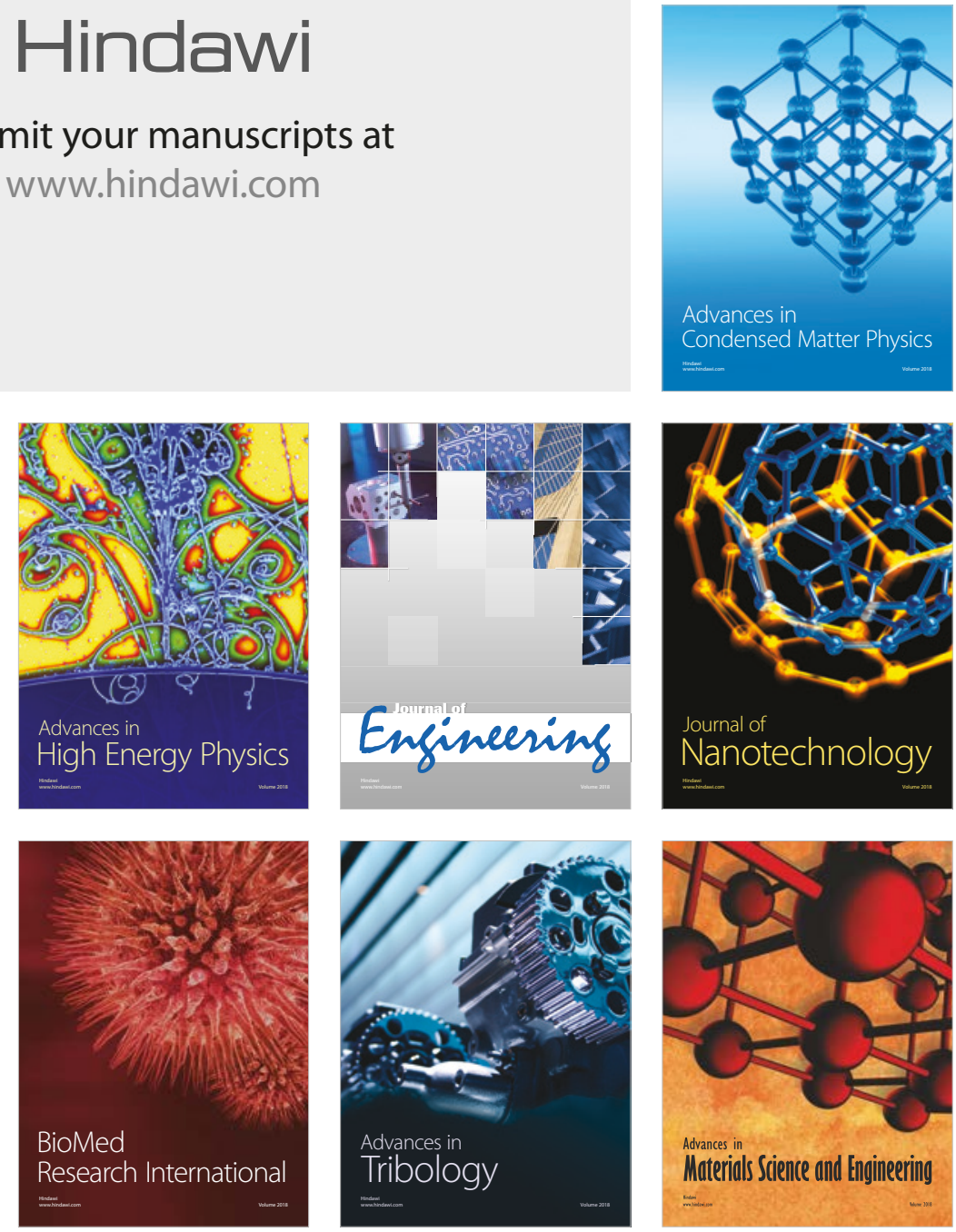